\title{
Editorial
}

Digestive

Diseases

\section{Rectal Cancer - Towards Establishing a New Strategy of Treatment}

\author{
Evaghelos Xynos ${ }^{\mathrm{a}}$ Christos Dervenis $^{\mathrm{b}}$ \\ ${ }^{a}$ Medical School, University of Crete, Heraklion, and b First Department of General Surgery, 'Agia Olga' Hospital, \\ Athens, Greece
}

During the last three decades, a great deal of progress has been achieved firstly in understanding genetics and natural history, secondly in the establishment of the diagnosis and the accurate staging, and thirdly in the therapeutic modalities of rectal cancer. That progress has led to a substantial improvement in survival and local recurrence rates, greater than that accomplished for colon cancer. Surgery still remains the cornerstone of rectal cancer treatment. The introduction of the concept of total mesorectal excision (TME) by Heald et al. [1], almost 25 years ago, resulted in local recurrence rates of less than $10 \%$ [2]. Further to adequate proximal and distal resection bowel margins, TME involves removal of the rectum along with the mesorectal fatty tissue that contains the regional lymph nodes and possible micrometastases covered by an intact rectal fascia propria. In recent years, there has been a debate on the exact anterior plane of dissection while performing a TME. Heald et al. [3] propose that the anterior plane of dissection should run in front of the Denonvilliers' fascia down to the point it fuses with the prostatic capsule, and not between the fascia and the anterior rectal wall. By this approach, which sounds reasonable in an anatomical and oncological sense, the anterior mesorectum of the middle third of the rectum covered by the Denonvilliers' fascia is removed. It is of great importance that both the surgeon

\section{KARGER}

Fax +4161306 1234

E-Mail karger@karger.ch

www.karger.com (c) 2007 S. Karger AG, Basel

0257-2753/07/0251-0005\$23.50/0

Accessible online at:

www.karger.com/ddi and the pathologist evaluate macroscopically and classify the completeness of the rectal cancer resection specimen. Quality control of surgery provides the first prognostic information concerning local and distant recurrence $[4,5]$.

With such impressive results of local control and survival after TME, adjuvant radiotherapy and chemotherapy seem to offer no additional benefit. However, there are always patients at risk to develop local recurrence and distant metastases. These subsets include patients with lymph node metastases, venous or lymphatic vessels invasion, distally located tumors most likely requiring an abdominoperineal resection (APR) and, most importantly, a threatened by tumor extension circumferential margin of resection (CRM). It is established beyond any doubt that the status of the CRM is possibly the strongest predictive factor of local recurrence or even survival. A positive CRM, namely presence of tumor at a distance $<1 \mathrm{~mm}$ from the CRM on the fixed specimen, is associated with local recurrence rates significantly greater than those seen in patients with negative CRM $[4,6,7]$. To improve surgical treatment outcomes in patients with extensive rectal tumors, neoadjuvant radiotherapy has been applied in order to downstage the disease. According to the Dutch Colorectal Cancer Group randomized trial [8], hypofractionated $5 \mathrm{~Gy} /$ day for 5 days radiother- 
apy followed by TME 7-10 days later offers local recurrence rates well below 5\% as compared to TME alone. The main message of the trial is that a multimodality therapeutic approach depending on the stage of the disease would optimize the results of TME. Several studies [9-12] propose the preoperative application of a full radiation scheme of 45-60 Gy over a period of 5 weeks, concomitantly accompanied by chemotherapy, and followed by TME approximately 10 weeks later. According to the results of those studies, neoadjuvant chemoradiation not only downstages but also downsizes the disease, rendering feasible an $\mathrm{R} 0$ resection of an otherwise locally extended and possibly not resectable rectal tumor, offering better local recurrence and survival rates and possibly preserving the anal sphincters. However, there are still numerous questions seeking answers, questions concerning the optimal tailoring of the neoadjuvant treatment according to stage and histological characteristics of the tumor, the optimal imaging modality of final staging prior to surgery, the time interval between end of chemoradiation and surgery, the histopathological assessment of the primary tumor, lymph nodes and CRM on the fixed surgical specimen, and the functional outcome. Furthermore, under the light of neoadjuvant treatment, the place of adjuvant chemotherapy and additional radiotherapy should be redefined. The CR07 multicentric randomized trial, which has been recently completed, attempts to answer some of the aforementioned questions. According to the results of the trial, administration of neoadjuvant short course radiotherapy followed by TME is associated with significantly lower local recurrence and higher survival rates compared to TME alone that is followed by adjuvant chemoradiotherapy only in T3, T4 and node-positive cases. This stands true for all rectal tumors including $\mathrm{T} 1$ and $\mathrm{T} 2$ as well as tumors located in the proximal-endoperitoneal third of the rectum [pers. commun.].

It is conceivable that preoperative staging is of paramount importance for the staging of the disease and, in particular, for assessing extension of the primary tumor and status of regional lymph nodes. Imaging modalities, including magnetic resonance imaging, spiral computed tomography and endoanal ultrasonography, complement each other to offer pretreatment accurate staging rates well over $90 \%$ [13]. Furthermore, it is imperative that specific imaging definitions must be further elaborated so as to improve staging of the rectal cancer after neoadjuvant chemoradiotherapy.

There is nowadays a trend towards performing more sphincter-preserving operations than APR for tumors located in the lower third of the rectum. All trials reported that radiotherapy should be given prior to surgery, in order to reduce the stage and the size of the tumor [14-16]. Some authors [17] even recommend neoadjuvant long-course chemoradiotherapy even for stage I tumors, although there are reports according to which, despite downsizing, neoadjuvant long-course chemoradiotherapy does not seem to increase the sphincter-preserving operation rate compared to preoperative shortcourse radiation for T3-T4 low rectal tumors [18]. However, the concept that tumor downsizing and increase in the macroscopic distance between the lower edge of the tumor and the anorectal ring facilitates low resections and anastomoses may be hazardous; there might be microscopic tumor deposits distal to the macroscopic lower edge of the tumor and a compromise in distal clearance of the rectal wall, thus increasing the risk of anastomotic recurrence. Therefore, it is recommended that the decision to perform a sphincter-sparing operation should be taken prior to the neoadjuvant treatment. Depending on the type of resection, conventional low anterior or intersphincteric, the colo-anal anastomosis is fashioned either with the use of stapling devices or by hand-sawing. Intersphincteric resection may be applied to tumors located almost even in the anal canal, provided the sphincters are not invaded. In this case the colon in a form of a pouch is anastomosed by hand to the anoderm [19].

Although more sphincter-preserving operations are attempted, APR is still applied, apparently following new specific indications. An increased risk of local and distant recurrence after an APR compared to low anterior resection of the rectum has been reported. This is attributed to the low level and the unfavorable characteristics of the tumor, as well as the increased incidence of positive CRM, rather than the type of the operation itself. Related to the depth of invasion, very low rectal tumors tend to spread laterally and downwards to the lateral pelvic wall lymph nodes. Hence, in this case the criterion of a free CRM is not satisfied by TME only. In other words, a resection specimen involving the whole mesorectum and the anal sphincters creating a sort of 'waist' in between them is not of adequate quality. On the high-quality specimen after an APR, there should appear a part of the rectosacral fascia with the mesorectum above and the plate of the lavatory muscles below, in the form of a 'cylinder' $[20,21]$. At present however, there are no strong data supporting the view that a 'cylindrical' APR specimen is associated with less recurrence and better survival rates than the conventional 
one in the form of a 'waist'. In any case, it is suggested that low rectal tumors should be treated rather aggressively by adding preoperative chemoradiation more often than in higher rectal tumors.

Recently, surgery for rectal cancer can also be accomplished by laparoscopy. At present, the results published show that the approach is safe in terms of postoperative morbidity and mortality. The quality of the specimen is comparable to that after conventional surgery. Because of the better view into the pelvis, there is a feeling among the surgeons practicing laparoscopy that a $\mathrm{R} 0$ resection for rectal cancer is easier, safer and more complete by laparoscopy than by the open approach. However, strong evidence concerning local recurrence and survival is still awaited [22]. Several technical problems, such us proper distal transection of the rectum in obese male patients, also have to be resolved by improvement of instrumentation.

For rectal tumors with minimal invasion of the bowel wall, local excision by means of the transanal endoscopic microsurgery (TEM) device has been attempted. T1 tumors are usually excised by this approach, although there is a report of TEM for T2 rectal tumors after a short course of preoperative radiotherapy with a local recur- rence rate of $5 \%$. However, data derived from some series show that TEM, even for T1 tumor, fails to match the good results acquired after abdominal surgery, in terms of recurrence [23]. Possibly, a multimodality approach, including neoadjuvant treatment, may improve the longterm results of TEM.

Several ongoing trials aim towards offering plausible answers to most of the above questions and setting new guidelines for a multimodality treatment of rectal cancer with even further improved recurrence and survival rates and quality of life. Furthermore, multidisciplinary approach requires not only close collaboration between the surgeon, the pathologist, the radiologist and the oncologist, but also core training of the team.

In the present issue of Digestive Disease, most of the current knowledge and trends in the treatment of rectal cancer is gathered and presented in reviews. Also, in a review of the molecular biological changes characterizing colorectal cancer, the possible implementations of genetic studies in screening and follow-up is discussed. Finally, the issue includes original articles which focus on several aspects of the management of patients with colorectal cancer.

\section{References}

1 Heald RJ, Husband EM, Ryall RDH: The mesorectum in rectal cancer surgery - the clue to pelvic recurrence? Br J Surg 1982;69:613616.

2 MacFarlane JK, Ryall RDH, Heald RJ: Mesorectal excision for rectal cancer. Lancet 1993; 341:457-460.

3 Heald RJ, Moran BJ, Brown G, Daniels IR: Optimal total mesorectal excision for rectal cancer is by dissection in front of Denonvillier's fascia. Br J Surg 2004;91:121-123.

4 Nagtegaal ID, van de Velde CJH, van der Worp E, Kapiteijn E, Quirke P, van Krieken JHJM, Dutch Colorectal Cancer Group: Macroscopic evaluation of the rectal cancer resection specimen: clinical significance of the pathologist in quality control. J Clin Oncol 2002;20:1729-1734.

5 Quirke P: Training and quality assurance for rectal cancer: 20 years of data is enough. Lancet (Oncol) 2003;4:695-702.

6 Nagtegaal ID, Marijnen CA, Kranenbarg EK: Circumferential margins involvement is still an important predictor of local recurrence in rectal carcinoma: not one millimeter but two millimeters is the limit. Am J Surg Pathol 2002;26:350-357.
7 Wibe A, Rendedal PR, Svensson E: Prognostic significance of the circumferential resection margin following total mesorectal excision for rectal cancer. Br J Surg 2002;89: 327-334.

8 Kapiteijn E, Marijnen CA, Nagtegaal ID: Preoperative radiotherapy combined with total mesorectal excision for resectable rectal cancer. N Engl J Med 2001;345:638-646.

9 Rodel C, Marus P, Papadopoulos T, et al: Prognostic significance of tumour regression after preoperative chemoradiotherapy for rectal cancer. J Clin Oncol 2005;23:86888696.

10 Chan AK, Wong A, Jenken D, et al: Posttreatment TNM staging is a prognostic indicator of survival and recurrence in tethered or fixed rectal carcinoma after preoperative chemotherapy and radiotherapy. Int J Radiat Oncol Biol Phys 2005;1: 665-677.

11 Pucciarelli S, Toppan P, Friso ML, et al: Complete pathologic response following preoperative chemoradiation therapy for middle to lower rectal cancer is not a prognostic factor for better outcome. Dis Colon Rectum 2004;47:798-807.
12 Bossett JF, Calsis G, Mineur L, et al: Enhanced tumouricidal effect of chemotherapy with preoperative radiotherapy for rectal cancer. Preliminary results EORTC 22921. J Clin Oncol 2005;24:5620-5627.

13 Brown G, Radcliffe AG, Newcombe RG, et al: Preoperative assessment of prognostic factors in rectal cancer using high-resolution magnetic resonance imaging. Br J Surg 2003; 90:355-364.

14 Crane CH, Skibber JM, Feig BW, et al: Response to preoperative chemoradiation increases the use of sphincter-preserving surgery in patients with locally advanced low rectal carcinoma. Cancer 2003;97:517-524.

15 Kuvshinoff B, Maghfoor I, Miedema B, et al: Distal margin requirements after operative chemoradiotherapy for distal rectal carcinomas: are $<$ or $=1 \mathrm{~cm}$ distal margin sufficient? Ann Surg Oncol 2000;8:163-169.

16 Rouanet P, Saint-Aubert B, Lemansky B, et al: Restorative and nonrestorative surgery for low rectal carcinoma after high-dose radiation. Dis Colon Rectum 2002;45:305315 . 
17 Mohiuddin M, Regine WF, Marks GJ, Marks JW: High-dose preoperative radiation and the challenge of sphincter-preservation surgery for cancer of the distal $2 \mathrm{~cm}$ of the rectum. Int J Radiat Oncol Biol Phys 1998;40: 569-574.

18 Bujko K, Nowacki MP, Nasierowska-Guttmejer A, et al: Sphincter preservation following preoperative radiotherapy for rectal cancer: report of a randomized trial comparing short-term radiotherapy vs. conventionally fractionated radiochemotherapy. Radiother Oncol 2004;72:15-24.
19 Rullier E, Goffe B, Bonnel C, Zerbib F, Caudri M, Saric J: Preoperative radiochemotherapy and sphincter-saving resection for T3 carcinomas of the lower third of the rectum. Ann Surg 2001;234:633-640.

20 Wibe A, Syse A, Andersen E, Tretli S, Myrvold $\mathrm{HE}$, Soreide $\mathrm{O}$ on behalf of the Norwegian Rectal Cancer Group: Oncological outcomes after total mesorectal excision for cure for cancer of the lower rectum: anterior vs. abdominoperineal resection. Dis Colon Rectum 2004;47:48-58.

21 Radcliffe A: Can the results of anorectal (abdominoperineal resection) be improved: are circumferential resection margins too often positive? Colorectal Dis 2006;8:160-167.
22 Guillou PJ, Quirke P, Thorpe H, et al.: Shortterm endpoints of conventional versus laparoscopic assisted surgery in patients with colorectal cancer (MRC CLASICC trial): multicentre, randomised controlled trial. Lancet 2005;365:1718-1726.

23 Lezoche E, Guerrieri M, Paganini AM, et al: Transanal endoscopic versus total mesorectal laparoscopic resections of T2-N0 low rectal cancers after neoadjuvant treatment: a prospective randomized trial with a 3 -year minimum follow-up period. Surg Endosc 2005;19:751-756. 